\title{
Analysis on Exponential Stability of Hybrid Pantograph Stochastic Differential Equations with Highly Nonlinear Coefficients
}

\author{
Surong $\mathrm{You}^{\mathrm{a}, *}$, Wei $\mathrm{Mao}^{\mathrm{b}}$, Xuerong $\mathrm{Mao}^{\mathrm{c}}$, Liangjian $\mathrm{Hu}^{\mathrm{a}}$ \\ ${ }^{a}$ College of Science \\ Donghua University, Shanghai 201620, China \\ ${ }^{b}$ School of Mathematics and Information Technology \\ Jiangsu Second Normal University, Nanjing 210013, China \\ ${ }^{c}$ Department of Mathematics and Statistics \\ University of Strathclyde, Glasgow G1 1XH, U.K.
}

\begin{abstract}
This paper discusses exponential stability of solutions for highly nonlinear hybrid pantograph stochastic differential equations(PSDEs). Two criteria are proposed to guarantee exponential stability of the solution. The first criterion is a Khasminskii-type condition involving general Lyapunov functions. The second is developed on coefficients of the equation in virtue of M-matrix techniques. Based on the second criterion, robust stability of a perturbed hybrid PSDE is also investigated. The theory shows how much an exponentially stable hybrid PSDE can tolerate to remain stable.
\end{abstract}

Key words: Brownian motion, Markov chain, hybrid pantograph stochastic differential equations, exponential stability, generalized Itô formula, robust stability.

\section{Introduction}

Stochastic differential delay equations (SDDEs) are widely used to model those systems dependent on the present and past states(see, e.g. [1]-[8]). When these systems experience abrupt changes in their structures and parameters, continuous-time Markov chains are introduced to form SDDEs with Markovian switching, also known as hybrid SDDEs.

One of the important issues in the study of hybrid SDDEs is the automatic control, with current emphasis placed on asymptotic stability and boundedness arising from automatic control. There is an intensive literature in this area and we mention, for example, [9]-[13]. In particular, [9] and [11] are two of most cited papers while [12] is the first book in this area. In most of the above mentioned references, coefficients of those systems are assumed to satisfy local Lipschitz condition and linear growth condition. However, the linear growth condition is usually violated in many practical applications. There have been some papers discussing existence, uniqueness and stability of solutions of highly nonlinear SDDEs, for example, [14]-[17]. Recently, [18] discussed asymptotic stability and boundedness of solutions to nonlinear hybrid SDDEs with constant delays or differentiable bounded variable delays. Also in [19],

\footnotetext{
*Corresponding author. Tel: +86 67792086-556; Fax: +86 67792085

Email addresses: sryou@dhu.edu.cn (Surong You), mwzy365@126.com (Wei Mao), x.mao@strath.ac.uk (Xuerong Mao), ljhu@dhu.edu.cn (Liangjian $\mathrm{Hu}$ )
} 
robust exponential stability and boundedness of highly nonlinear hybrid SDDEs with constant delays were investigated.

Hybrid pantograph stochastic differential equations(PSDEs) are special SDDEs that have unbounded delays (see e.g.[20],[21]). PSDEs have been frequently applied in many practical areas, such as mechanic, biology, engineering and finance. The existence-uniqueness theorem of the solution for a linear PSDE was established in [20]. On stability of a PSDE, [1] investigated the growth and decay rates of special scalar PSDEs, where equations had linear drifts with unbounded delays and diffusions without delays. [18] proposed a Khasminskii-type condition for a nonlinear hybrid PSDE, under which the polynomial stability of the solution could be derived. [22] extended the condition of [18] to the case that different types of functions or polynomials with different orders occured in the Lyapunov operator. [23] investigated the exponential stability of a class of hybrid PSDE, where the coefficients were dominated by polynomials with high orders. Almost sure exponential stability of both exact and numerical solutions could be derived under such conditions. But we argue that the criteria proposed in [23] were independent on the transition matrix, so that the system would be stable at any mode. This paper will apply the technique in [19] to get exponential stability of a PSDE under suitable conditions. Compared to [23], in our result, the transition matrix of Markovian switching will play an important rule in the criterion. Also M-matrix techniques will be used to form an efficient criterion. We will show exponential stability in the $p$ th moment and almost sure exponential stability under the same condition.

When studying asymptotic properties, robust analyses on stability and boundedness have received a great deal of attention. On SDDEs, [24] and [25] discussed robust stability of linear delay equations. [26] studied robust stochastic stability of a linear system. In [27], robust stability of uncertain linear or semilinear SDDEs had been discussed. The robust stability of a stochastic delay interval system with Markovian switching was studied in [11]. Recently in [19], the robust stability and boundedness of hybrid SDDEs with constant delay and high nonlinearity had been well treated. In this paper, after giving an efficient criterion to evaluate the exponential stability of PSDEs, robust analysis on exponential stability will also be discussed. Applying the theory, we can discuss how much the perturbation can be in order for a perturbed system remaining stable.

This article is arranged as follows. A general criterion including Lyapunov functions is proposed in section 2, under which the PSDE system will be asymptotically bounded or exponentially stable. In section 3, an efficient criterion with the aid of M-matrices will be discussed. Robust analyses on boundedness and exponential stability are developed in section 4 . Some examples are discussed to illustrate the theory in section 5 and conclusions are made in section 6 .

\section{General results}

Throughout this paper, we use following notations. Let $\left(\Omega, \mathcal{F},\left\{\mathcal{F}_{t}\right\}_{t \geq 0}, P\right)$ be a complete probability space with the filtration $\left\{\mathcal{F}_{t}\right\}_{t \geq 0}$ satisfying the usual conditions (i.e. it is increasing and right continuous with $\mathcal{F}_{0}$ containing all $P$-null sets). Let $B(t)=\left(B_{1}(t), \cdots, B_{m}(t)\right)^{T}$ be an $m$-dimensional Brownian motion defined on the probability space. Let $|\cdot|$ be the Euclidean norm in $\mathbb{R}^{n}$. If $A$ is a vector or matrix, 
its transpose is denoted by $A^{T}$. If $A$ is a matrix, its trace norm is denoted by $|A|=\sqrt{\operatorname{trace}\left(A^{T} A\right)}$. Let $\mathbb{R}_{+}=[0, \infty)$.

Let $r(t), t \geq 0$, be a right-continuous Markov chain on the probability space taking values in a finite state space $S=\{1,2, \cdots, N\}$ with generator $\Gamma=\left(\gamma_{i j}\right)_{N \times N}$ given by

$$
P\{r(t+\Delta)=j \mid r(t)=i\}=\left\{\begin{aligned}
\gamma_{i j} \Delta+o(\Delta) & \text { if } i \neq j \\
1+\gamma_{i i} \Delta+o(\Delta) & \text { if } i=j
\end{aligned}\right.
$$

with $\Delta>0 . \gamma_{i j} \geq 0$ is the transition rate from $i$ to $j$ if $i \neq j$, while $\gamma_{i i}=-\sum_{j \neq i} \gamma_{i j}$. Assume that the Markov chain $r(\cdot)$ is independent of the Brownian motion $B(\cdot)$.

Denote by $C\left(\mathbb{R}^{n} \times \mathbb{R}_{+} ; \mathbb{R}_{+}\right)$the family of continuous functions from $\mathbb{R}^{n} \times \mathbb{R}_{+}$to $\mathbb{R}_{+}$, also by $C^{2,1}\left(\mathbb{R}^{n} \times\right.$ $\left.\mathbb{R}_{+} \times S ; \mathbb{R}_{+}\right)$the family of continuous functions $V(x, t, i)$ from $\mathbb{R}^{n} \times \mathbb{R}_{+} \times S$ to $\mathbb{R}_{+}$, such that for each $i \in S, V(x, t, i)$ is continuously twice differentiable in $x$ and once in $t$.

Consider a hybrid pantograph stochastic differential equation

$$
d x(t)=f(x(t), x(\theta t), t, r(t)) d t+g(x(t), x(\theta t), t, r(t)) d B(t)
$$

with $0<\theta<1$. Due to its special feature, we only need to know the initial data

$$
x(0)=x_{0} \in \mathbb{R}^{n} \text { and } r(0)=i_{0} \in S
$$

in order to solve the equation.

The well-known conditions imposed for the existence and uniqueness of the global solution are the local Lipschitz condition and the linear growth condition (see e.g. [4]-[8]). Let us state the local Lipschitz condition.

Assumption 2.1. For each integer $h \geq 1$, there exists a constant $K_{h}>0$ such that

$$
|f(x, y, t, i)-f(\bar{x}, \bar{y}, t, i)| \vee|g(x, y, t, i)-g(\bar{x}, \bar{y}, t, i)| \leq K_{h}(|x-\bar{x}|+|y-\bar{y}|)
$$

holds for those $x, y, \bar{x}, \bar{y} \in \mathbb{R}^{n}$ with $|x| \vee|\bar{x}| \vee|y| \vee|\bar{y}| \leq h$ and any $(t, i) \in \mathbb{R}_{+} \times S$.

However we will replace the linear growth condition by a more general condition, a KhasminskiiType condition as applied in [18],[19],[22],[23], to guarantee the existence of a unique global solution. Also this condition will lead to exponential stability of the solution. Before stating the general condition, we give one more notation. Given $V(x, t, r(t)) \in C^{2,1}\left(\mathbb{R}^{n} \times \mathbb{R}_{+} \times S, \mathbb{R}_{+}\right)$, we define the function $L V$ : $\mathbb{R}^{n} \times \mathbb{R}^{n} \times \mathbb{R}_{+} \times S \rightarrow \mathbb{R}$ by

$$
\begin{aligned}
L V(x, y, t, i) & =V_{t}(x, t, i)+V_{x}(x, t, i) f(x, y, t, i) \\
& +\frac{1}{2} \operatorname{trace}\left[g^{T}(x, y, t, i) V_{x x}(x, t, i) g(x, y, t, i)\right]+\sum_{j=1}^{N} \gamma_{i j} V(x, t, j),
\end{aligned}
$$

where $V_{t}(x, t, i)=\frac{\partial V(x, t, i)}{\partial t}, V_{x}(x, t, i)=\left(\frac{\partial V(x, t, i)}{\partial x_{1}}, \cdots, \frac{\partial V(x, t, i)}{\partial x_{n}}\right)^{T}$ and $V_{x x}=\left(\frac{\partial^{2} V(x, t, i)}{\partial x_{k} \partial x_{l}}\right)_{n \times n}$. Let us emphasize that $L V$ is defined on $\mathbb{R}^{n} \times \mathbb{R}^{n} \times \mathbb{R}_{+} \times S$ while $V$ on $\mathbb{R}^{n} \times \mathbb{R}_{+} \times S$. 
Assumption 2.2. There exist three functions $V(x, t, r(t)) \in C^{2,1}\left(\mathbb{R}^{n} \times \mathbb{R}_{+} \times S, \mathbb{R}_{+}\right), U_{1}(x, t), U_{2}(x, t) \in$ $C\left(\mathbb{R}^{n} \times \mathbb{R}_{+}, \mathbb{R}_{+}\right)$, and positive constants $c_{1}, c_{2}, \alpha_{i}, i=1, \cdots, 5$ such that

$$
\begin{gathered}
\lim _{|x| \rightarrow \infty}\left(\inf _{t \geq 0} U_{1}(x, t)\right)=\infty, \\
c_{1} U_{1}(x, t) \leq V(x, t, i) \leq c_{2} U_{1}(x, t), \quad \text { for any }(x, t, i) \in \mathbb{R}^{n} \times \mathbb{R}_{+} \times S, \\
L V(x, y, t, i) \leq \alpha_{1}-\alpha_{2} U_{1}(x, t)+\alpha_{3} \theta \exp (-(1-\theta) t) U_{1}(y, \theta t)-\alpha_{4} U_{2}(x, t)+\alpha_{5} \theta \exp (-(1-\theta) t) U_{2}(y, \theta t), \\
\text { where } c_{1}>c_{2}, \alpha_{2}>\alpha_{3}, \alpha_{4}>\alpha_{5} .
\end{gathered}
$$

In above assumption, there are two auxiliary functions $U_{1}(x, t), U_{2}(x, t)$ used to dominate $L V$. We can see that if $U_{2}(x, t)$ has a lower order of infinity than $U_{1}(x, t)$ as $|x| \rightarrow \infty, L V$ will have the same order as $V$. The main objective of this paper is to investigate the setting that $L V$ may have higher order than $V$ or $U_{1}$, due to high nonlinearity of the equation. So in this paper, we will focus on the situation that $U_{2}(x, t)$ has a higher order of infinity than $U_{1}(x, t)$, for example $U_{1}(x, t)=|x|^{2}$ and $U_{2}(x, t)=|x|^{4}$. Also the factor $\exp (-(1-\theta) t)$ is important for exponential stability of the solution under further conditions as depicted in Theorem 2.4. Just as discussed in [18], if there is no $\exp (-(1-\theta) t)$, we can only get the polynomial stability of the system. Also it can be argued that if $\hat{U}_{2}$ is set as $\hat{U}_{2}=U_{1}+U_{2}$, equations (4) and (5) can be rewritten as $c_{1} U_{1}(x, t) \leq V(x, t, i) \leq c_{2} \hat{U}_{2}(x, t)$ and

$$
L V(x, y, t, i) \leq \alpha_{1}-\left(\alpha_{2} \wedge \alpha_{4}\right) \hat{U}_{2}(x, t)+\left(\alpha_{3} \vee \alpha_{5}\right) \theta \exp (-(1-\theta) t) \hat{U}_{2}(y, \theta t)
$$

which is a strong form of Assumption 2.2 in [18]. The reason why we separate $\hat{U}_{2}$ into $U_{1}$ and $U_{2}$ is for clarity of upcoming discussion in section 3 , where $U_{1}(x, t)$ and $U_{2}(x, t)$ are chosen as $|x|^{p}$ and $|x|^{q}$, respectively.

Theorem 2.3. Under Assumption 2.1 and 2.2, following statements are true.

(1) For any initial data (2), there exists a unique global solution $x(t)$ to the system $(1)$ on $[0, \infty)$.

(2) For $t>0$,

$$
\int_{0}^{t} E U_{2}(x(s), s) d s \leq \frac{c_{2}}{\alpha_{4}-\alpha_{5}} U_{1}(x(0), 0)+\frac{\alpha_{1} t}{\alpha_{4}-\alpha_{5}}
$$

(3) The solution is asymptotically bounded in the sense of

$$
\limsup _{t \rightarrow \infty} E U_{1}(x(t), t) \leq \frac{\alpha_{1}}{\varepsilon c_{1}}
$$

where $\varepsilon$ is defined by

$$
\varepsilon:=\min \left(1,\left(\alpha_{2}-\alpha_{3}\right) / c_{2}\right)
$$

Proof. (1) The existence and uniqueness of the solution can be got easily by the same method used in Theorem 3.1 of [18] or in Theorem 2.5 of [19]. Here we omit its proof in order to concentrate exponential stability of the solution.

(2) Let $k_{0}>0$ be large enough for $\left|x_{0}\right|<k_{0}$. For each integer $k \geq k_{0}$, define the stopping time

$$
\tau_{k}=\inf \{t|| x(t) \mid \leq k\}, k=1,2, \cdots
$$


where throughout this paper we set $\inf \emptyset=\infty$ (as usual $\emptyset$ denotes the empty set). By the generalized Itô formula (see e.g. [12]), we obtain that for $t \geq 0$,

$$
E\left(V\left(x\left(t \wedge \tau_{k}\right), t \wedge \tau_{k}, r\left(t \wedge \tau_{k}\right)\right)\right)=V(x(0), 0, r(0))+E \int_{0}^{t \wedge \tau_{k}} L V(x(s), x(\theta s), s, r(s)) d s .
$$

Applying condition (4), (5) and $\exp (-(1-\theta) t)<1$, it can be got that

$$
\begin{aligned}
& c_{1} E\left(U_{1}\left(x\left(t \wedge \tau_{k}\right), t \wedge \tau_{k}\right)\right) \\
\leq \quad & c_{2} U_{1}(x(0), 0)+\alpha_{1} t-\alpha_{2} E \int_{0}^{t \wedge \tau_{k}} U_{1}(x(s), s) d s+\alpha_{3} \theta E \int_{0}^{t \wedge \tau_{k}} U_{1}(x(\theta s), \theta s) d s \\
& -\alpha_{4} E \int_{0}^{t \wedge \tau_{k}} U_{2}(x(s), s) d s+\alpha_{5} \theta E \int_{0}^{t \wedge \tau_{k}} U_{2}(x(\theta s), \theta s) d s .
\end{aligned}
$$

Obviously, we have

$$
\alpha_{3} \theta E \int_{0}^{t \wedge \tau_{k}} U_{1}(x(\theta s), \theta s) d s=\alpha_{3} E \int_{0}^{\theta\left(t \wedge \tau_{k}\right)} U_{1}(x(s), s) d s \leq \alpha_{3} E \int_{0}^{t \wedge \tau_{k}} U_{1}(x(s), s) d s,
$$

and similarly

$$
\alpha_{5} \theta E \int_{0}^{t \wedge \tau_{k}} U_{2}(x(\theta s), \theta s) d s \leq \alpha_{5} E \int_{0}^{t \wedge \tau_{k}} U_{2}(x(s), s) d s .
$$

Consequently, from $\alpha_{2}>\alpha_{3}$ and $\alpha_{4}>\alpha_{5},(10)$ gives $c_{1} E\left(U_{1}\left(x\left(t \wedge \tau_{k}\right), t \wedge \tau_{k}\right)\right) \leq c_{2} U_{1}(x(0), 0)+\alpha_{1} t$. Let $k \rightarrow \infty$ and then get $c_{1} E\left(U_{1}(x(t), t)\right) \leq c_{2} U_{1}(x(0), 0)+\alpha_{1} t$. Meanwhile, we also have

$$
0 \leq c_{1} E\left(U_{1}\left(x\left(t \wedge \tau_{k}\right), t \wedge \tau_{k}\right)\right) \leq c_{2} U_{1}(x(0), 0)+\alpha_{1} t-\left(\alpha_{4}-\alpha_{5}\right) E \int_{0}^{t \wedge \tau_{k}} U_{2}(x(s), s) d s,
$$

which can be arranged to

$$
E \int_{0}^{t \wedge \tau_{k}} U_{2}(x(s), s) d s \leq \frac{c_{2}}{\alpha_{4}-\alpha_{5}} U_{1}(x(0), 0)+\frac{\alpha_{1} t}{\alpha_{4}-\alpha_{5}} .
$$

Now (6) is derived by letting $k \rightarrow \infty$ and using Fubini's theorem.

(3) Let $\tau_{k}$ the same stopping time defined in (9). Applying the generalized Itô formula on function $\exp (\varepsilon t) V(x(t), t, r(t))$, we obtain that for $t \geq 0$,

$$
\begin{aligned}
& c_{1} E\left(\exp \left(\varepsilon\left(t \wedge \tau_{k}\right)\right) U_{1}\left(x\left(t \wedge \tau_{k}\right), t \wedge \tau_{k}\right)\right) \\
\leq \quad & c_{2} U_{1}(x(0), 0)+E \int_{0}^{t \wedge \tau_{k}} \varepsilon \exp (\varepsilon s) V(x(s), s) d s+E \int_{0}^{t \wedge \tau_{k}} \exp (\varepsilon s) L V(x(s), x(\theta s), s, r(s)) d s .
\end{aligned}
$$

It can be calculated that

$$
E \int_{0}^{t \wedge \tau_{k}} \varepsilon \exp (\varepsilon s) V(x(s), s) d s \leq c_{2} \varepsilon E \int_{0}^{t \wedge \tau_{k}} \exp (\varepsilon s) U_{1}(x(s), s) d s,
$$

and

$$
\begin{aligned}
& E \int_{0}^{t \wedge \tau_{k}} \exp (\varepsilon s) L V(x(s), x(\theta s), s, r(s)) d s \\
\leq & E \int_{0}^{t \wedge \tau_{k}} \exp (\varepsilon s)\left[\alpha_{1}-\alpha_{2} U_{1}(x(s), s)+\alpha_{3} \theta \exp (-(1-\theta) s) U_{1}(x(\theta s), \theta s)\right. \\
& \left.-\alpha_{4} U_{2}(x(s), s)+\alpha_{5} \theta \exp (-(1-\theta) s) U_{2}(x(\theta s), \theta s)\right] d s \\
= & \alpha_{1} E \int_{0}^{t \wedge \tau_{k}} \exp (\varepsilon s) d s-\alpha_{2} E \int_{0}^{t \wedge \tau_{k}} \exp (\varepsilon s) U_{1}(x(s), s) d s+\alpha_{3} \theta E \int_{0}^{t \wedge \tau_{k}} \exp ((\varepsilon-1+\theta) s) U_{1}(x(\theta s), \theta s) d s \\
& -\alpha_{4} E \int_{0}^{t \wedge \tau_{k}} \exp (\varepsilon s) U_{2}(x(s), s) d s+\alpha_{5} \theta E \int_{0}^{t \wedge \tau_{k}} \exp ((\varepsilon-1+\theta) s) U_{2}(x(\theta s), \theta s) d s .
\end{aligned}
$$

But $\alpha_{1} E \int_{0}^{t \wedge \tau_{k}} \exp (\varepsilon s) d s \leq\left(\alpha_{1} / \varepsilon\right) \exp (\varepsilon t)$, and

$$
\alpha_{3} \theta E \int_{0}^{t \wedge \tau_{k}} \exp ((\varepsilon-1+\theta) s) U_{1}(x(\theta s), \theta s) d s=\alpha_{3} E \int_{0}^{\theta\left(t \wedge \tau_{k}\right)} \exp \left(\frac{\varepsilon-1+\theta}{\theta} u\right) U_{1}(x(u), u) d u .
$$


By $0<\theta<1$ and $\varepsilon \leq 1$, we can get $(\varepsilon-1+\theta) / \theta \leq \varepsilon$ easily, so that

$$
\alpha_{3} \theta E \int_{0}^{t \wedge \tau_{k}} \exp ((\varepsilon-1+\theta) s) U_{1}(x(\theta s), \theta s) d s \leq \alpha_{3} E \int_{0}^{t \wedge \tau_{k}} \exp (\varepsilon s) U_{1}(x(s), s) d s
$$

and similarly,

$$
\alpha_{5} \theta E \int_{0}^{t \wedge \tau_{k}} \exp ((\varepsilon-1+\theta) s) U_{2}(x(\theta s), \theta s) d s \leq \alpha_{5} E \int_{0}^{t \wedge \tau_{k}} \exp (\varepsilon s) U_{2}(x(s), s) d s .
$$

Substituting above inequalities into equations (12) and (13), We will have

$$
\begin{aligned}
& c_{1} E\left(\exp \left(\varepsilon\left(t \wedge \tau_{k}\right)\right) U_{1}\left(x\left(t \wedge \tau_{k}\right), t \wedge \tau_{k}\right)\right) \\
\leq & c_{2} U_{1}(x(0), 0)+\frac{\alpha_{1}}{\varepsilon} \exp (\varepsilon t)-\left(\alpha_{2}-\varepsilon c_{2}-\alpha_{3}\right) E \int_{0}^{t \wedge \tau_{k}} \exp (\varepsilon s) U_{1}(x(s), s) d s \\
& -\left(\alpha_{4}-\alpha_{5}\right) E \int_{0}^{t \wedge \tau_{k}} \exp (\varepsilon s) U_{2}(x(s), s) d s .
\end{aligned}
$$

From $\alpha_{4}>\alpha_{5}$ and $\varepsilon \leq\left(\alpha_{2}-\alpha_{3}\right) / c_{2}$, we deduce

$$
E\left(\exp \left(\varepsilon\left(t \wedge \tau_{k}\right)\right) U_{1}\left(x\left(t \wedge \tau_{k}\right), t \wedge \tau_{k}\right)\right) \leq \frac{c_{2}}{c_{1}} U_{1}(x(0), 0)+\frac{\alpha_{1}}{c_{1} \varepsilon} \exp (\varepsilon t) .
$$

Letting $k \rightarrow \infty$, we have

$$
\exp (\varepsilon t) E U_{1}(x(t), t) \leq \frac{c_{2}}{c_{1}} U_{1}(x(0), 0)+\frac{\alpha_{1}}{c_{1} \varepsilon} \exp (\varepsilon t),
$$

or equivalently,

$$
E U_{1}(x(t), t) \leq \frac{c_{2}}{c_{1}} U_{1}(x(0), 0) \exp (-\varepsilon t)+\frac{\alpha_{1}}{c_{1} \varepsilon}
$$

which yields

$$
\limsup _{t \rightarrow \infty} E U_{1}(x(t), t) \leq \frac{\alpha_{1}}{c_{1} \varepsilon}
$$

as required.

This theorem gives asymptotic boundedness in terms of $U_{1}$ and $U_{2}$. If we know, for example, $U_{1}(x, t) \geq|x|^{p}$, the solution of equation (1) will be asymptotically bounded in the $p$-th moment. To get exponential stability of the system, we need one additional condition, $\alpha_{1}=0$, as illustrated in following theorem.

Theorem 2.4. Let Assumption 2.1 and 2.2 hold with $\alpha_{1}=0$ in (5), the solution is also exponential stability as

$$
\limsup _{t \rightarrow \infty} \frac{1}{t} \log E U_{1}(x(t), t) \leq-\varepsilon
$$

and

$$
\limsup _{t \rightarrow \infty} \frac{1}{t} \log U_{1}(x(t), t) \leq-\varepsilon,
$$

where $\varepsilon$ is defined as in (8). Meanwhile, the solution satisfies

$$
\int_{0}^{\infty} E U_{2}(x(s), s) d s<\infty .
$$

Proof. If $\alpha_{1}=0$, equation (16) turns to be $E U_{1}(x(t), t) \leq\left(c_{2} / c_{1}\right) U_{1}(x(0), 0) \exp (-\varepsilon t)$, which just means the statement (17). 
From (11) with $\alpha_{1}=0$, we see $E \int_{0}^{t \wedge \tau_{k}} U_{2}(x(s), s) d s \leq \frac{c_{2}}{\alpha_{4}-\alpha_{5}} U_{1}(x(0), 0)$. Taking $k \rightarrow \infty$ and then $t \rightarrow \infty$, Fubini's theorem gives

$$
\int_{0}^{\infty} E U_{2}(x(s), s) d s \leq \frac{c_{2}}{\alpha_{4}-\alpha_{5}} U_{1}(x(0), 0)<\infty
$$

To show almost sure exponential stability, we apply the generalized Itô formula on $\exp (\varepsilon t) V(x(t), t, r(t))$ to get

$\exp (\varepsilon t) V(x(t), t, r(t))=V(x(0), 0, r(0))+\int_{0}^{t} \exp (\varepsilon s)[\varepsilon V(x(s), s, r(s))+L V(x(s), x(\theta s), s, r(s))] d s+M(t)$,

where $M(t)$ is a local martingale with the initial value $M(0)=0$.

As $\alpha_{1}=0$, applying the same argument on deriving (14) and the definition of $\varepsilon$, we also have

$$
\begin{aligned}
& \int_{0}^{t} \exp (\varepsilon s)[\varepsilon V(x(s), s, r(s))+L V(x(s), x(\theta s), s, r(s))] d s \\
\leq & -\left(\alpha_{2}-\varepsilon c_{2}-\alpha_{3}\right) \int_{0}^{t} \exp (\varepsilon s) U_{1}(x(s), s) d s-\left(\alpha_{4}-\alpha_{5}\right) \int_{0}^{t} \exp (\varepsilon s) U_{2}(x(s), s) d s \\
\leq & 0 .
\end{aligned}
$$

Then applying condition (4) to equation (20), we get

$$
c_{1} \exp (\varepsilon t) U_{1}(x(t), t) \leq c_{2} U_{1}(x(0), t)+M(t)
$$

Applying the nonnegative semi-martingale convergence theorem(see for example, [12]), we obtain that $\limsup _{t \rightarrow \infty} c_{1} \exp (\varepsilon t) U_{1}(x(t), t)<\infty, a . s$. from the inequality $(21)$. So there exists a finite positive random variable $\eta$ such that $\sup _{0 \leq t<\infty} c_{1} \exp (\varepsilon t) U_{1}(x(t), t) \leq \eta, a . s .$, which just implies $(18)$ as required.

Just as discussed after Theorem 2.3, if $U_{1}(x, t) \geq|x|^{p},(17)$ and (18) show the exponential stability in the sense of the $p$-th moment and almost sure. While if $U_{2}(x, t) \geq|x|^{q}$, the solution will be $H_{\infty}$-stable, because $\int_{0}^{\infty} E|x(t)|^{q} d t<\infty$ is valid from (19).

\section{Further Criteria in Terms of M-matrices}

In this section, we will establish further criteria on exponential stability of equation (1) in terms of M-matrices. We will choose $V(x, t, i)=\theta_{i}|x|^{p}$ for suitable $\theta_{i}>0, U_{1}(x)=|x|^{p}$ and $U_{2}(x)=|x|^{q}$

in Assumption 2.2. With these special functions, we can see direct requirements on the structure of coefficients $f, g$ in equation (1) in order to guarantee exponential stability of its solution. The technique used here comes from [19], where M-matrices are used to check exponential stability of SDDEs with constant delays. We are lucky to see that similar criteria can be used to guarantee the exponential stability of PSDEs.

For the convenience of the reader, let us cite some useful results on M-matrices. For more detailed information please see e.g.[12]. First we give some notations. If $B$ is a vector or matrix, $B \gg 0$ means that all elements of $B$ are positive. If $B_{1}$ and $B_{2}$ are two vectors of matrices with same dimensions, we write $B_{1} \gg B_{2}$ if and only if $B_{1}-B_{2} \gg 0$. We adopt the traditional notation by letting

$$
Z^{N \times N}=\left\{A=\left(a_{i j}\right)_{N \times N}: a_{i j} \leq 0, i \neq j\right\} .
$$


Definition 3.1. A square matrix $A=\left(a_{i j}\right)_{N \times N}$ is called a nonsingular M-matrix if $A$ can be expressed in the form $A=s I-B$ with $s>\rho(B)$ while all elements of $B$ are nonnegative, where $I$ is the identity matrix and $\rho(B)$ the spectral radius of $B$.

There are many conditions which are equivalent to the statement that $A$ is a nonsingular M-matrix and we cite some of them for the use of this paper(see e.g.[12]).

Lemma 3.2. If $A \in Z^{N \times N}$, then following statements are equivalent:

(1) A is a nonsingular M-matrix.

(2) A is semi-positive; that is, there exists $x \gg 0$ in $\mathbb{R}^{N}$ such that $A x \gg 0$.

(3) $A^{-1}$ exists and its elements are all nonnegative.

(4) All the leading principal minors of $A$ are positive; that is

$$
\left|\begin{array}{ccc}
a_{11} & \cdots & a_{i k} \\
\vdots & \cdots & \vdots \\
a_{k 1} & \cdots & a_{k k}
\end{array}\right|>0, \text { for every } k=1,2, \cdots, N .
$$

Now we state the hypothesis on coefficients $f, g$, in term of an M-matrix.

Assumption 3.3. Given $q>p \geq 2$, assume that for any $i \in S$, there exists non-negative constants $\beta_{i 1}, \beta_{i 3}, \beta_{i 4}, \beta_{i 5}$ and a real number $\beta_{i 2}$, such that

$$
\begin{gathered}
x^{T} f(x, y, t, i)+\frac{p-1}{2}|g(x, y, t, i)|^{2} \\
\leq \beta_{i 1}+\beta_{i 2}|x|^{2}+\beta_{i 3} \theta \exp \left(-\frac{2}{p}(1-\theta) t\right)|y|^{2}-\beta_{i 4}|x|^{q-p+2}+\beta_{i 5} \theta \exp \left(-\frac{q-p+2}{q}(1-\theta) t\right)|y|^{q-p+2}
\end{gathered}
$$

for all $(x, y, t) \in \mathbb{R}^{n} \times \mathbb{R}^{n} \times \mathbb{R}_{+}$, and

$$
\mathcal{A}:=-\operatorname{diag}\left(p \beta_{12}, \cdots, p \beta_{N 2}\right)-\Gamma
$$

is a nonsingular M-matrix.

We remark that there is a similar condition as (22) in [19]:

$$
x^{T} f(x, y, t, i)+\frac{p-1}{2}|g(x, y, t, i)|^{2} \leq \beta_{i 1}+\beta_{i 2}|x|^{2}+\beta_{i 3}|y|^{2}-\beta_{i 4}|x|^{q-p+2}+\beta_{i 5}|y|^{q-p+2},
$$

which leads to exponential stability of a SDDE with constant delay. If we impose this assumption on a PSDE, we can only get polynomial stability of its solution, just as discussed in [18]. We will see that two terms involving exponential decay are necessary to get the exponential stability of a PSDE.

By properties of M-matrices as in 3.2, we have a vector with all positive entries defined by the nonsingular M-matrix $\mathcal{A}$ :

$$
\left(\theta_{1}, \cdots, \theta_{N}\right)^{T}:=\mathcal{A}^{-1} \overrightarrow{\mathbf{1}} \gg 0
$$

where $\overrightarrow{\mathbf{1}}=(1, \cdots, 1)^{T}$.

Theorem 3.4. Let Assumption 2.1 and 3.3 hold. $\theta_{i}, i \in S$ is defined from (24). Set $c_{1}=\min _{i \in S} \theta_{i}$, $c_{2}=\max _{i \in S} \theta_{i} . \delta_{1}=\max _{i \in S} p \theta_{i} \beta_{i 1}, \delta_{3}=\max _{i \in S} p \theta_{i} \beta_{i 3}, \delta_{4}=\min _{i \in S} p \theta_{i} \beta_{i 4}, \delta_{5}=\max _{i \in S} p \theta_{i} \beta_{i 5}$, and $\delta=\left(1-\delta_{3}\right) / 2$. 
Assume that

$$
\delta_{3} \leq 1 \text { and } \delta_{4} \geq \delta_{5}
$$

Then for any given initial data (2), there is a unique global solution $x(t)$ to the hybrid PSDE (1) on $[0, \infty)$. Moreover, the solution is asymptotically bounded in the pth moment, that is, the solution satisfies

$$
\limsup _{t \rightarrow \infty} E|x(t)|^{p} \leq \frac{\alpha_{1}}{\varepsilon c_{1}},
$$

and

$$
\int_{0}^{t} E|x(s)|^{q} d s \leq \frac{c_{2}}{\alpha_{4}-\alpha_{5}}|x(0)|^{p}+\frac{\alpha_{1} t}{\alpha_{4}-\alpha_{5}},
$$

where $\varepsilon=\min \left(1,\left(\alpha_{2}-\alpha_{3}\right) / c_{2}\right)$.

In above results, $\alpha_{1} \sim \alpha_{5}$ are defined as

$$
\alpha_{1}=\frac{2}{p} \delta^{-\frac{p-2}{2}} \delta_{1}^{\frac{p}{2}}, \alpha_{2}=1-\frac{p-2}{p}\left(\delta+\delta_{3} \theta\right), \alpha_{3}=\frac{2}{p} \delta_{3}, \alpha_{4}=\delta_{4}-\delta_{5} \theta \frac{p-2}{q}, \text { and } \alpha_{5}=\delta_{5} \frac{q-p+2}{q} .
$$

Proof. The proof is an application of Theorem 2.3. We will use $U_{1}(x, t)=|x|^{p}$ and $U_{2}(x, t)=|x|^{q}$ in equation (5). The function $V: \mathbb{R}^{n} \times \mathbb{R}_{+} \times S \rightarrow \mathbb{R}_{+}$is defined by $V(x, t, r(t))=\theta_{r(t)}|x|^{p}$. We recall that from the definition of $\theta, \mathcal{A} \theta=\overrightarrow{\mathbf{1}}$, or equivalently, for any $i \in S, p \beta_{i 2} \theta_{i}+\sum_{j=1}^{N} \gamma_{i j} \theta_{j}=-1$.

We see for any $i \in S, c_{1}|x|^{p} \leq V(x, t, i) \leq c_{2}|x|^{p}$. Now we compute $L V(x, y, t, i)$. For any $i \in S$,

$$
\begin{aligned}
& L V(x, y, t, i) \\
= & p \theta_{i}|x|^{p-2} x^{T} f(x, y, t, i)+\frac{p(p-2)}{2} \theta_{i}|x|^{p-4}\left|x^{T} g(x, y, t, i)\right|^{2}+\frac{1}{2} p \theta_{i}|x|^{p-2}|g|^{2}+\sum_{j=1}^{N} \gamma_{i j} \theta_{j}|x|^{p} \\
\leq & p \theta_{i}|x|^{p-2}\left[x^{T} f(x, y, t, i)+\frac{p-1}{2}|g(x, y, t, i)|^{2}\right]+\sum_{j=1}^{N} \gamma_{i j} \theta_{j}|x|^{p} \\
\leq & p \theta_{i} \beta_{i 1}|x|^{p-2}+\left(p \theta_{i} \beta_{i 2}+\sum_{j=1}^{N} \gamma_{i j} \theta_{j}\right)|x|^{p}+p \theta_{i} \beta_{i 3} \theta \exp \left(-\frac{2}{p}(1-\theta) t\right)|x|^{p-2}|y|^{2} \\
& -p \theta_{i} \beta_{i 4}|x|^{q}+p \theta_{i} \beta_{i 5} \theta \exp \left(-\frac{q-p+2}{q}(1-\theta) t\right)|x|^{p-2}|y|^{q-p+2} .
\end{aligned}
$$

By the fundamental inequality $a^{\alpha} b^{1-\alpha} \leq \alpha a+(1-\alpha) b$, for $a, b \geq 0$, and $\alpha \in[0,1]$, we have

$$
\exp \left(-\frac{2}{p}(1-\theta) t\right)|x|^{p-2}|y|^{2} \leq \frac{p-2}{p}|x|^{p}+\frac{2}{p} \exp (-(1-\theta) t)|y|^{p},
$$

and

$$
\exp \left(-\frac{q-p+2}{q}(1-\theta) t\right)|x|^{p-2}|y|^{q-p+2} \leq \frac{p-2}{q}|x|^{q}+\frac{q-p+2}{q} \exp (-(1-\theta) t)|y|^{q} .
$$

Also for any $i \in S$,

$$
p \theta_{i} \beta_{i 1}|x|^{p-2} \leq \delta_{1}|x|^{p-2}=\left(\delta^{-\frac{p-2}{2}} \delta_{1}^{\frac{p}{2}}\right)^{\frac{2}{p}}\left(\delta|x|^{p}\right)^{\frac{p-2}{p}} \leq \frac{2}{p} \delta^{-\frac{p-2}{2}} \delta_{1}^{\frac{p}{2}}+\frac{\delta(p-2)}{p}|x|^{p} .
$$

Substituting above inequalities into (29), we obtain

$$
L V(x, y, t, i) \leq \alpha_{1}-\alpha_{2}|x|^{p}+\alpha_{3} \theta \exp (-(1-\theta) t)|y|^{p}-\alpha_{4}|x|^{q}+\alpha_{5} \theta \exp (-(1-\theta) t)|y|^{q},
$$

with the definition of $\alpha_{1} \sim \alpha_{5}$ given in (28).

Also from $0<\theta<1, \delta_{3} \leq 1$ and $\delta_{4} \geq \delta_{5}$, we can see that

$$
\alpha_{2}-\alpha_{3}=\frac{1}{p}\left[\frac{p+2}{2}-\left(\frac{6-p}{2}+(p-2) \theta\right) \delta_{3}\right]>\frac{p+2}{2 p}\left(1-\delta_{3}\right) \geq 0
$$


and

$$
\alpha_{4}-\alpha_{5}=\left(\delta_{4}-\delta_{5}\right)+\frac{p-2}{q} \delta_{5}(1-\theta)>0 .
$$

It is now clear that Assumption 3.3 and condition (25) produce a special case as in Assumption 2.2 with $U_{1}(x, t)=|x|^{p}, U_{2}(x, t)=|x|^{q}$ and all coefficients defined as in the premise of this theorem. And then (26) and (27) can be obtained from (6) and (7) in Theorem 2.3, respectively.

The following theorem gives a criterion on both exponential stability and $H_{\infty}$-stability.

Theorem 3.5. Let Assumptions 2.1 and 3.3 hold with $\beta_{i 1}=0$ for all $i \in S$. For any given initial data $(2)$, the unique global solution $x(t)$ has the properties that

$$
\limsup _{t \rightarrow \infty} \frac{1}{t} \log \left(E|x(t)|^{p}\right) \leq-\varepsilon
$$

and

$$
\limsup _{t \rightarrow \infty} \frac{1}{t} \log (|x(t)|) \leq-\frac{\varepsilon}{p}, \text { a.s. }
$$

where $\varepsilon$ is defined as in Theorem 3.4. Moreover, $x(t)$ is $H_{\infty}$-stable:

$$
\int_{0}^{\infty} E|x(t)|^{q} d t<\infty
$$

Proof. If for all $i \in S, \beta_{i 1}=0, \alpha_{1}$ should be 0 . The results in Theorem 2.4 are valid for $U_{1}(x, t)=$ $|x|^{p}, U_{2}(x, t)=|x|^{q}$ and $\varepsilon=\left(\alpha_{2}-\alpha_{3}\right) / c_{2}$, where $c_{2}, \alpha_{2}$ and $\alpha_{3}$ are defined as in Theorem 3.4. And then (30)-(32) come from (17)-(19), respectively.

\section{Robust Exponential Stability Analysis of PSDEs}

In this section, we discuss two robust exponential stability problems in PSDEs. The first problem is based on following $n$-dimensional nonlinear hybrid differential equation

$$
d x(t)=f(x(t), t, r(t)) d t,
$$

where $f: \mathbb{R}^{n} \times \mathbb{R} \times S \rightarrow \mathbb{R}$ satisfies the local Lipschitz condition and similar condition as in Assumption 3.3. Let us make it clear.

Assumption 4.1. Let $q>p \geq 2$ and assume that for each $i \in S$, there are nonnegative numbers $\bar{\beta}_{i 1}, \bar{\beta}_{i 4}$ and a real number $\bar{\beta}_{i 2}$ such that for all $(x, t) \in \mathbb{R}^{n} \times \mathbb{R}_{+}$,

$$
x^{T} f(x, t, i) \leq \bar{\beta}_{i 1}+\bar{\beta}_{i 2}|x|^{2}-\bar{\beta}_{i 4}|x|^{q-p+2},
$$

and $\overline{\mathcal{A}}:=-\operatorname{diag}\left(p \bar{\beta}_{12}, \cdots, p \bar{\beta}_{N 2}\right)-\Gamma$ is a nonsingular $M$-matrix.

Under above assumption, equation (33) is asymptotically bounded in the $p$-th moment according to Theorem 3.4. Now if there is a stochastic delay perturbation in the diffusion part as

$$
d x(t)=f(x(t), t, r(t)) d t+G(x(\theta t), t, r(t)) d B(t),
$$

will the system remain stable under suitable conditions on $G$ ? In order that equation (35) has a solution, we assume that $G$ satisfies the local Lipschitz condition. Furthermore, we need another condition on $G$. 
Assumption 4.2. Let $q>p \geq 2$ be the same as in Assumption 4.1 and assume that for each $i \in S$, there are nonnegative functions $\bar{\beta}_{i 3}(t), \bar{\beta}_{i 5}(t)$ and number $\bar{\beta}_{i 6} \geq 0$, such that for any $(y, t)$,

$$
|G(y, t, i)|^{2} \leq \bar{\beta}_{i 6}+\bar{\beta}_{i 3}(t)|y|^{2}+\bar{\beta}_{i 5}(t)|y|^{q-p+2} .
$$

Now we want to discuss the requirements on $\bar{\beta}_{i 6}, \bar{\beta}_{i 3}(t)$ and $\bar{\beta}_{i 5}(t)$ such that the perturbed system is asymptotically bounded or exponentially stable. Answers are just applications of Theorem 3.4 and 3.5 as stated in following two theorems.

Theorem 4.3. Let Assumption 4.1 and 4.2 hold. Define

$$
\left(\bar{\theta}_{1}, \cdots, \bar{\theta}_{N}\right)^{T}=\overline{\mathcal{A}}^{-1} \overrightarrow{1} \gg 0
$$

If for any $i \in S$,

$$
\bar{\beta}_{i 3}(t) \leq \frac{2 \theta}{p(p-1) \bar{\theta}_{i}} \exp \left(-\frac{2}{p}(1-\theta) t\right)
$$

and

$$
\bar{\beta}_{i 5}(t) \leq \frac{2 \theta}{(p-1) \bar{\theta}_{i}}\left(\min _{j} \bar{\theta}_{j} \bar{\beta}_{j 4}\right) \exp \left(-\frac{q-p+2}{q}(1-\theta) t\right),
$$

then the perturbed system (35) is asymptotically bounded in the pth moment.

Proof. Combining (34) and (36) together, we can easily obtain

$$
x^{T} f+\frac{p-1}{2}|g|^{2} \leq\left(\bar{\beta}_{i 1}+\frac{p-1}{2} \bar{\beta}_{i 6}\right)+\bar{\beta}_{i 2}|x|^{2}+\frac{p-1}{2} \bar{\beta}_{i 3}(t)|y|^{2}-\bar{\beta}_{i 4}|x|^{q-p+2}+\frac{p-1}{2} \bar{\beta}_{i 5}(t)|y|^{q-p+2} .
$$

Then by (38) and (39), it is just

$$
\begin{gathered}
x^{T} f+\frac{p-1}{2}|g|^{2} \leq\left(\bar{\beta}_{i 1}+\frac{p-1}{2} \bar{\beta}_{i 6}\right)+\bar{\beta}_{i 2}|x|^{2}+\frac{\theta}{p \bar{\theta}_{i}} \exp \left(-\frac{2}{p}(1-\theta) t\right)|y|^{2} \\
-\bar{\beta}_{i 4}|x|^{q-p+2}+\frac{\theta}{\bar{\theta}_{i}}\left(\min _{j} \bar{\theta}_{j} \bar{\beta}_{j 4}\right) \exp \left(-\frac{q-p+2}{q}(1-\theta) t\right)|y|^{q-p+2} .
\end{gathered}
$$

Now it is easy to check that condition (25) in Theorem 3.4 is satisfied with $\delta_{3}=1, \delta_{4}=\delta_{5}=$ $\min _{j} \bar{\theta}_{j} \bar{\beta}_{j 4}$. The perturbed system (35) is proved to be asymptotically bounded in the $p$ th moment as required.

Just as differences between Theorem 3.4 and 3.5, we also have the similar result.

Theorem 4.4. Let conditions (34) and (36) hold, with $\bar{\theta}_{i}$ defined in (37). If Assumption 4.1 and 4.2 hold with $\bar{\beta}_{i 1}=\bar{\beta}_{i 6}=0$ for all $i \in S$, the perturbed system (35) is not only exponentially stable in the pth moment, but also almost sure exponentially stable. Moreover, the solution of the perturbed system is $H_{\infty}$ stable in the sense

$$
\int_{0}^{\infty} E|x(t)|^{q} d t<\infty
$$

In the second problem, we consider the case that a general SDE

$$
d x(t)=f(x(t), t, r(t)) d t+g(x(t), t, r(t)) d B(t)
$$

has a delay perturbation in the drift part, which takes the form of

$$
d x(t)=[f(x(t), t, r(t))+F(x(t), x(\theta t), t, r(t))] d t+g(x(t), t, r(t)) d B(t) .
$$


As a standing hypothesis, we assume that both $f, g$ and $F$ satisfy the local Lipschitz condition. Also in order that the original system (40) is bounded or stable, we should impose some conditions on $f$ and $g$. For the ease of further discussion, we impose two assumptions on $f, g$ and $F$.

Assumption 4.5. Let $q>p \geq 2$. Assume that for each $i \in S$, there exists nonnegative numbers $\hat{\beta}_{i 1}, \hat{\beta}_{i 4}$, a real number $\hat{\beta}_{i 2}$ and a positive number $\tilde{\beta}_{i 2}$, such that for all $(x, t)$,

$$
x^{T} f(x, t, i)+\frac{p-1}{2}|g(x, t, i)|^{2} \leq \hat{\beta}_{i 1}+\left(\hat{\beta}_{i 2}-\tilde{\beta}_{i 2}\right)|x|^{2}-\hat{\beta}_{i 4}|x|^{q-p+2}
$$

and $\hat{\mathcal{A}}:=-\operatorname{diag}\left(p \hat{\beta}_{12}, \cdots, p \hat{\beta}_{N 2}\right)-\Gamma$ is a nonsingular M-matrix.

Obviously, the original system (40) is asymptotically bounded, or exponentially stable as $\hat{\beta}_{i 1}=0, i \in$ $S$ under Assumption 4.5.

Assumption 4.6. Assume that for each $i \in S$, there are nonnegative functions $\eta_{i 3}(t), \eta_{i 5}(t)$ and nonnegative numbers $\eta_{i 1}, \eta_{i 2}, \eta_{i 4}$, such that for any $(x, y, t, i)$,

$$
|F(x, y, t, i)|^{2} \leq \eta_{i 1}+\eta_{i 2}|x|^{2}+\eta_{i 3}(t)|y|^{2}+\eta_{i 4}|x|^{q-p+2}+\eta_{i 5}(t)|y|^{q-p+2} .
$$

Theorem 4.7. Let Assumption 4.5 and 4.6 hold. Define

$$
\left(\hat{\theta}_{1}, \cdots, \hat{\theta}_{N}\right)^{T}=\hat{\mathcal{A}}^{-1} \overrightarrow{1} \gg 0 .
$$

If for every $i \in S$,

$$
\begin{gathered}
\eta_{i 2} \leq \tilde{\beta}_{i 2}^{2} \\
\eta_{i 3}(t) \leq \frac{2 \tilde{\beta}_{i 2} \theta}{\hat{\theta}_{i}} \exp \left(-\frac{2}{p}(1-\theta) t\right), \\
\eta_{i 4} \leq 2 \tilde{\beta}_{i 2} \hat{\beta}_{i 4}
\end{gathered}
$$

and

$$
\eta_{i 5}(t) \leq \frac{2 \tilde{\beta}_{i 2} \theta}{\hat{\theta}_{i}}\left(\min _{j \in S} \hat{\theta}_{j}\left(\hat{\beta}_{j 4}-\frac{\eta_{j 4}}{2 \tilde{\beta}_{j 2}}\right)\right) \exp \left(-\frac{q-p+2}{q}(1-\theta) t\right),
$$

then the perturbed system (41) is asymptotically bounded in the pth moment.

Proof. We only need to show that under conditions (45)-(48), the coefficients $f, g, F$ satisfy the requirement in Theorem 3.4.

Firstly, we can compute that

$$
\begin{aligned}
& x^{T} F(x, y, t, i) \leq|x||F(x, y, t, i)| \leq \frac{\tilde{\beta}_{i 2}}{2}|x|^{2}+\frac{1}{2 \tilde{\beta}_{i 2}}|F(x, y, t, i)|^{2} \\
\leq \quad & \frac{\eta_{i 1}}{2 \tilde{\beta}_{i 2}}+\left(\frac{\tilde{\beta}_{i 2}}{2}+\frac{\eta_{i 2}}{2 \tilde{\beta}_{i 2}}\right)|x|^{2}+\frac{\eta_{i 3}(t)}{2 \tilde{\beta}_{i 2}}|y|^{2}+\frac{\eta_{i 4}}{2 \tilde{\beta}_{i 2}}|x|^{q-p+2}+\frac{\eta_{i 5}(t)}{2 \tilde{\beta}_{i 2}}|y|^{q-p+2} .
\end{aligned}
$$

Applying (45)-(48), coefficients in perturbed system (41) satisfy

$$
\begin{aligned}
& x^{T}(f+F)+\frac{p-1}{2}|g|^{2} \\
\leq & \left(\hat{\beta}_{i 1}+\frac{\eta_{i 1}}{2 \tilde{\beta}_{i 2}}\right)+\left(\hat{\beta}_{i 2}-\frac{\tilde{\beta}_{i 2}}{2}+\frac{\eta_{i 2}}{2 \hat{\beta}_{i 2}}\right)|x|^{2}+\frac{\eta_{i 3}(t)}{2 \hat{\beta}_{i 2}}|y|^{2}-\left(\hat{\beta}_{i 4}-\frac{\eta_{i 4}}{2 \hat{\beta}_{i 2}}\right)|x|^{q-p+2}+\frac{\eta_{i 5}(t)}{2 \hat{\beta}_{i 2}}|y|^{q-p+2} \\
\leq & a_{i 1}+a_{i 2}|x|^{2}+a_{i 3} \theta \exp \left(-\frac{2}{p}(1-\theta) t\right)|y|^{2}-a_{i 4}|x|^{q-p+2}+a_{i 5} \theta \exp \left(-\frac{q-p+2}{q}(1-\theta) t\right)|y|^{q-p+2}
\end{aligned}
$$

where

$$
a_{i 1}=\hat{\beta}_{i 1}+\frac{\eta_{i 1}}{2 \tilde{\beta}_{i 2}}, a_{i 2}=\hat{\beta}_{i 2}, a_{i 3}=\frac{1}{\hat{\theta}_{i}}, a_{i 4}=\hat{\beta}_{i 4}-\frac{\eta_{i 4}}{2 \tilde{\beta}_{i 2}}, a_{i 5}=\frac{1}{\hat{\theta}_{i}}\left(\min _{j \in S} \hat{\theta}_{j} a_{j 4}\right) .
$$

Now we can see that all requirements in Theorem 3.4 are obtained, so that the solution of perturbed system (41) is asymptotically bounded in the $p$ th moment. 
Moreover, we can also get following result on robust exponential stability by virtue of Theorem 3.5.

Theorem 4.8. Let conditions (45)-(48) hold with $\hat{\theta}_{i}$ defined in (44). If Assumption 4.5 and 4.6 hold with $\hat{\beta}_{i 1}=\eta_{i 1}=0$ for all $i \in S$, the perturbed system (41) is not only exponentially stable in the pth moment, but also almost sure exponentially stable. Moreover, the solution of the perturbed system is $H_{\infty}$ stable: $\int_{0}^{\infty} E|x(t)|^{q} d t<\infty$.

\section{Examples}

In this section, we will give two examples to illustrate our theory. The first example is to check the stability of a given PSDE, while the second one will cover robust analysis. In two examples, $B(t)$ is fixed as a scalar Brownian motion, which is independent on the Markov chain appeared in two systems. We only give the one dimensional hybrid PSDEs in order to avoid complicate calculations.

Example 1. Consider following hybrid PSDE

$$
d x(t)=f(x(t), t, r(t)) d t+g(x(0.1 t), t, r(t)) d B(t)
$$

where $r(t)$ is a right-continuous Markov chain taking value in $S=\{1,2\}$ with the generator $\Gamma=$

$\left(\begin{array}{cc}-1 & 1 \\ 4 & -4\end{array}\right)$. The coefficients are $f(x, t, 1)=-x-2 x^{3}, g(y, t, 1)=0.5 y^{2} \exp (-0.45 t), f(x, t, 2)=x-x^{3}$, and $g(y, t, 2)=0.1 y \exp (-0.45 t)$.

Set $p=2, q=4$. It is easy to see $x f(x, t, 1)+\frac{1}{2} g^{2}(y, t, 1)=-x^{2}-2 x^{4}+\frac{1}{8} y^{4} \exp (-0.9 t)$, and $x f(x, t, 2)+\frac{1}{2} g^{2}(y, t, 2)=x^{2}-x^{4}+\frac{1}{200} y^{2} \exp (-0.9 t)$.

The quantities appeared in (22) are $\beta_{11}=0, \beta_{12}=-1, \beta_{13}=0, \beta_{14}=2, \beta_{15}=1.25 ; \beta_{21}=0, \beta_{22}=$ $1, \beta_{23}=1 / 20, \beta_{24}=1$, and $\beta_{25}=0$.

We see that the matrix $\mathcal{A}$ in (23) is $\mathcal{A}=\left(\begin{array}{cc}3 & -1 \\ -4 & 2\end{array}\right)$ with the inverse $\mathcal{A}^{-1}=\left(\begin{array}{ll}1 & 0.5 \\ 2 & 1.5\end{array}\right)$, which shows that $\mathcal{A}$ is a nonsingular M-matrix.

By the definition in (24), $\theta_{1}=1.5, \theta_{2}=3.5$. Direct calculation gives the quantities involved in Theorem 3.4: $c_{1}=1.5, c_{2}=3.5, \delta_{1}=0, \delta_{3}=0.35<1, \delta_{4}=6, \delta_{5}=3.75, \alpha_{2}=1$ and $\alpha_{3}=0.35$. So from the result in Theorem 3.5, the solution of system (49) is exponentially stable in the second moment with the moment Lyapunov index $\varepsilon=\min (1,(1-0.35) / 3.5)=0.1857$. Also the solution is almost sure exponentially stable $\limsup _{t \rightarrow \infty} \frac{1}{t} \log (|x(t)|) \leq-0.0929$ and $H_{\infty}$ stable $\int_{0}^{\infty} E|x(t)|^{4} d t<\infty$.

Example 2. Consider a hybrid differential equation

$$
d x(t)=f(x(t), t, r(t)) d t
$$

where $r(t)$ is a right-continuous Markov chain taking values in $S=\{1,2\}$ with generator $\Gamma=\left(\begin{array}{cc}-1 & 1 \\ 6 & -6\end{array}\right)$, and $f(x, t, 1)=-x-4 x^{7}, f(x, t, 2)=x-x^{7}$. As a special equation of (1) without delay and diffusion terms, it can be easily checked by Theorem 3.5 that (50) is exponentially stable in the second and third moments. 
Now if the system has a perturbation in the diffusion term as

$$
d x(t)=f(x(t), t, r(t)) d t+g(x(t), x(0.1 t), t, r(t)) d B(t)
$$

where $g(x, y, t, 1)=a_{1} y \exp (-b t)$ and $g(x, y, t, 2)=a_{2} x y^{2} \exp (-b t)$. We now want to discuss how much the unknown quantities $a_{1}, a_{2}$ and $b$ can be such that the perturbed system (51) is also exponentially stable in the second or third moment.

Case 1: $p=2, q=8$

Let $p=2, q=8$. It can be estimated that

$$
x f(x, t, 1)+\frac{1}{2} g^{2}(x, y, t, 1)=-x^{2}-4 x^{8}+\frac{1}{2} a_{1}^{2} y^{2} \exp (-2 b t)
$$

and

$$
\begin{gathered}
x f(x, t, 2)+\frac{1}{2} g^{2}(x, y, t, 2)=x^{2}-x^{8}+\frac{a_{2}^{2}}{2} x^{2} y^{4} \exp (-2 b t) \\
\leq x^{2}-x^{8}+\frac{1}{8} x^{4}+\frac{a_{2}^{4}}{2} \exp (-4 b t) y^{8} \leq \frac{13}{12} x^{2}-\frac{23}{24} x^{8}+\frac{a_{2}^{4}}{2} \exp (-4 b t) y^{8},
\end{gathered}
$$

where the inequality $x^{4} \leq 2 / 3 x^{2}+1 / 3 x^{8}$ has been used.

The matrix $\mathcal{A}$ will be $\mathcal{A}=\left(\begin{array}{cc}3 & -1 \\ -6 & 23 / 6\end{array}\right)$ with its inverse $\mathcal{A}^{-1}=\left(\begin{array}{cc}0.6970 & 0.1818 \\ 1.0909 & 0.5455\end{array}\right) \cdot \mathcal{A}$ is a nonsingular M-matrix.

Now careful calculation leads to following conditions on $a_{1}, a_{2}$ and $b$ which will guarantee the moment and almost sure exponential stability of the solution to perturbed system $(51): a_{1}^{2} \exp (-2 b t) \leq$ $0.1138 \exp (-0.9 t)$ and $a_{2}^{4} \exp (-4 b t) \leq 0.1917 \exp (-0.9 t)$. So it will be clear that $b \geq 0.225,\left|a_{1}\right| \leq 0.3373$ and $\left|a_{2}\right| \leq 0.6617$ are enough for the perturbed system to be exponentially stable in the second moment.

Case 2: $p=3, q=9$

In this case, we could estimate $x f(x, t, 1)+g^{2}(x, y, t, 1)=-x^{2}-4 x^{8}+a_{1}^{2} y^{2} \exp (-2 b t)$, and

$$
x f(x, t, 2)+g^{2}(x, y, t, 2)=x^{2}-x^{8}+a_{2}^{2} x^{2} y^{4} \exp (-2 b t) \leq \frac{4}{3} x^{2}-\frac{5}{6} x^{8}+\frac{a_{2}^{4}}{2} \exp (-4 b t) y^{8} .
$$

The matrix $\mathcal{A}$ will be $\mathcal{A}=\left(\begin{array}{cc}4 & -1 \\ -6 & 2\end{array}\right)$ with the inverse $\mathcal{A}^{-1}=\left(\begin{array}{cc}1 & 0.5 \\ 3 & 2\end{array}\right)$.

Following the same argument, we will have $a_{1}^{2} \exp (-2 b t) \leq 0.02222 \exp (-0.6 t)$ and $a_{2}^{4} \exp (-4 b t) \leq$ $0.1667 \exp (-0.8889 t)$, giving $\left|a_{1}\right| \leq 0.1491,\left|a_{2}\right| \leq 0.6389$ and $b \geq 0.3$. Under these conditions on $a_{1}, a_{2}$ and $b$, the perturbed system will be exponentially stable in the third moment.

\section{Conclusion}

In this article, we have discussed asymptotic boundedness and exponential stability of hybrid PSDEs with higher nonlinearity. We have established two criteria, one on general Lyaponov functions and the other on coefficients of the equation. In virtue of M-matrices, the criterion can be verified easily. Also our theory is used to investigate robust boundedness and exponential stability of perturbed systems. 


\section{Acknowledgement}

This paper is completed when the first and second authors were visiting the Department of Mathematics and Statistics, University of Strathclyde, whose hospitality is greatly appreciated. The authors would also like to thank the financial supports from the National Natural Science Foundation of China(11471071, 11401261), the Natural Science Foundation of Shanghai(14ZR1401200).

\section{References}

[1] J.A.D. Appleby, Decay and growth rates of solutions of scalar stochastic delay differential equations with unbounded delay and state dependent noise, Stoch. Dyn. 5(2005) 133-147.

[2] J.A.D. Appleby, D.W. Reynolds, Decay rates of solutions of linear stochastic Volterra equations, Electron. J. Probab. 13(2008) 922-943

[3] V. Kolmanovskii, A. Myshkis, Applied Theory of Functional Differential Equations, Kluwer Academic Publishers, 1992.

[4] X. Mao, Stability of Stochastic Differential Equations with Respect to Semimartingales, Longman Scientific and Technical, 1991.

[5] X. Mao, Exponential Stability of Stochastic Differential Equations, Marcel Dekker, 1994.

[6] X. Mao, Stochastic Differential Equations and Applications, 2nd Edition, Horwood Publishing, Chichester, 2007.

[7] B. Song, J. Park, Z. Wu, Y. Zhang, New results on delay-dependent stability analysis for neutral stochastic delay systems, J. Franklin Inst., 50(4)(2013) 840-852.

[8] S.-E.A. Mohammed, Stochastic Functional Differential Equations, Longman Scientific and Technical, 1986.

[9] X. Mao, Stability of stochastic differential equations with Markovian switching, Stoch. Proc. Appl. 79(1999) 45-67.

[10] X. Mao, A. Matasov, A.B. Piunovskiy, Stochastic differential delay equations with Markovian switching, Bernoulli. 6(2000) 73-90.

[11] X. Mao, Exponential stability of stochastic delay interval systems with Markovian switching, IEEE Trans. Autom. Control. 47(2002) 1604-1612.

[12] X. Mao, C. Yuan, Stochastic Differential Equations with Markovian Switching, Imperial College Press, 2006.

[13] B. Song, J. Park, Z. Wu, X. Li, New results on delay-dependent stability analysis and stabilization for stochastic time-delay systems, Int. J. Robust Nonlin Control, 24(16)(2014) 2546-2559. 
[14] Q. Luo, X. Mao, Y. Shen, New criteria on exponential stability of neutral stochastic differential delay equations, Syst. Control Lett. 55(2006) 826-834.

[15] X. Mao, Razumikhin type theorems on exponential stability of neutral stochastic functional differential equations, SIAM J. Math. Anal. 28(2)(1997) 389-401.

[16] X. Mao, M.J Rassias, Khasminskii-type theorems for stochastic differential delay equations, Stoch. Anal. Appl. 23(2005) 1045-1069.

[17] X. Mao, Numerical solutions of stochastic differential delay equations under the generalized Khasminskii-type conditions, Appl. Math. Comp. 217(2011) 5512-5524.

[18] L. Hu, X. Mao, Y. Shen, Stability and boundedness of nonlinear hybrid stochastic differential delay equations, Syst. Control Lett. 62(2013) 178-187.

[19] L. Hu, X. Mao, L. Zhang, Robust stability and boundedness of nonlinear hybrid stochastic differential delay equations, IEEE Trans. Automa. Control. 58(2013) 2319-2332.

[20] C.T.H. Baker, E. Buckwar, Continuous $\theta$-methods for the stochstic pantograph equations, Electron. Trans. Numer. Anal. 11(2000) 131-151.

[21] Y. Xiao, M. Song, M. Liu, Convergence and stability of the semi-implicit Euler method with variable stepsize for a linear stochastic pantograph differential equations, Int. J. Numer. Anal. Model. 8(2011) 214-225.

[22] M. Milošević, Existence, uniqueness, almost sure polynomial stability of solution to a class of highly nonlinear pantograph stochastic differential equations and the Euler-Maruyama approximation, Appl. Math. Comput. 237(2014) 672-685.

[23] S. Zhou, M. Xue, Exponential stability for nonlinear hybrid stochastic pantograph equations and numberical approximation, Acta Math. Sci. 34B(2014) 1254-1270.

[24] J.-H. Su. Further results on the robust stability of linear systems with a single time delay, Syst. Control Lett. 23(1994) 375-379.

[25] C.-L. Tseng, I.-K. Fong, J.-H. Su, Robust stability analysis for uncertain delay systems with output feedback controller, Syst. Control Lett. 23(1994) 271-278.

[26] U.G. Haussmann, Asymptotic stability of the linear Itô equation in infinite dimensions, J. Math. Anal. Appl. 65(1978) 219-235.

[27] X. Mao, N. Koroleva, A. Rodkina, Robust stability of uncertain stochastic differential delay equations, Syst. Control. Lett. 35(1998) 325-336. 